\title{
Biological significance of PinX1 telomerase inhibitor in esophageal carcinoma treatment
}

\author{
XIANG-KUI FAN ${ }^{1}$, RUI-HUA YAN ${ }^{2}$, XIANG-QUN GENG ${ }^{1}$, JING-SHAN LI ${ }^{1}$, \\ XIANG-MING $\mathrm{CHEN}^{1}$ and JIAN-ZHE LI ${ }^{1}$ \\ ${ }^{1}$ Department of Tumor Surgery, Tai'an Central Hospital; ${ }^{2}$ Coal Workers' Sanatorium of Taishan, Tai'an, Shandong 271000, P.R. China
}

Received April 21, 2015; Accepted May 11, 2016

DOI: $10.3892 /$ etm.2016.3561

\begin{abstract}
In the present study, to investigate the expression of PinX1 gene and its functional effects in human esophageal carcinoma (Eca)-109 cell line, expression vectors of human PinX1 (pEGFP-C3-PinX1) and its small interfering RNA (PinX1-FAM-siRNA) were constructed and transfected into Eca-109 cells using Lipofectamine 2000. Firstly, the mRNA expression level of PinX1 was examined using reverse transcription-polymerase chain reaction (RT-PCR). Once successful transfection was achieved, the effects on the mRNA level of human telomerase reverse transcriptase (hTERT), telomerase activity, cell proliferation and apoptosis were examined by semi-quantitative RT-PCR, stretch PCR, MTT assay and flow cytometry, respectively. Analysis of restriction and sequencing demonstrated that the recombining plasmids were successfully constructed. The results also indicated that transfection with pEGFP-C3-PinX1 and PinX1-FAM-siRNA into Eca-109 cells significantly increased PinX1 mRNA, decreased hTERT mRNA by $29.9 \%(\mathrm{P}<0.05)$, and significantly reduced telomerase activity $(\mathrm{P}<0.05)$, inhibited cell growth, and increased the cell apoptotic index from $19.27 \pm 0.76$ to $49.73 \pm 2 \%$. The transfected PinX1-FAM-SiRNA exhibited PinX1 mRNA expression levels that were significantly decreased by $70 \%(\mathrm{P}<0.05)$, whereas the remaining characteristics of Eca-109 cells, including cell growth, mRNA level of hTERT, telomerase activity and cell apoptotic index were not altered. Exogenous PinX1 has been demonstrated to be highly expressed in human Eca. PinX1 can inhibit human telomerase activity and the expression of hTERT mRNA, reduce tumor cell growth and induce apoptosis. Notably, these inhibitory functions were inhibited by silencing PinX1 in Eca with PinX1-FAM-siRNA. PinX1 was successfully increased and decreased in the present study, demonstrating that it may
\end{abstract}

Correspondence to: Professor Xiang-Kui Fan, Department of Tumor Surgery, Tai'an Central Hospital, 29 Long Tan Road, Tai'an, Shandong 271000, P.R. China

E-mail: fanxiangkui6345@163.com

Key words: esophageal carcinoma, PinX1 gene, siRNA, telomerase, apoptosis be a potential telomerase activity inhibitor. As PinX1 is an endogenous telomerase inhibitor, it may be used as a novel tumor-targeted gene therapy.

\section{Introduction}

Esophageal carcinoma (Eca) is a recurrent malignant neoplasm that is most common in China, resulting in China having the highest mortality and hospitalization rates for Eca. Eca is complicated by various causes, genes and phases; therefore, the majority of patients are diagnosed during the middle or late stages of the cancer. Furthermore, the prognosis is not promising with a 5-year survival rate of 5-20\% (1). Therefore, the mechanism underlying Eca must be explored in order to provide effective treatment. Previous studies have demonstrated that antisense oligodeoxynucleotides are able to inhibit tumor cell growth by inhibiting RNA as well as human telomerase reverse transcriptase (hTERT) $(2,3)$. In Eca, the amount of positive RNA is as high as $90.8 \%$, whereas normal esophagus smooth muscle cells are negative (4). RNA-targeted genetic treatment is a popular novel treatment for Eca. Pin 2/TRF 1 interacting protein 1 (PinX1) has been demonstrated to be a potential endogenous telomerase inhibitor that is able to inhibit the telomerase activity of some cancer cells', resulting in apoptosis $(5,6)$. However, this remains controversial as other studies have reported opposite findings $(7,8)$. PinX1 may function via different mechanisms in different tumors, and the mechanisms underlying the inhibition of the telomerase or RNA are complex. In order to investigate PinX1's inhibiting mechanism in Eca and telomerase activity, expression vectors of human PinX1 (pEGFP-C3-PinX1) and its small interfering RNA (PinX1-FAM-siRNA) were constructed. The present study investigates whether PinX1 may be a potential telomerase activity inhibitor, and whether it can be used as a novel tumor-targeted gene therapy.

\section{Materials and methods}

Materials. Austrian newborn cow blood serum was purchased from Weijia Company (Guangzhou, China); Lipofectamine 2000 and all primers (Invitrogen; Thermo Fisher Scientific, Inc., Waltham, MA, USA); reverse transcription-polymerase chain reaction (RT-PCR) detection kit (Takara Biotechnology Co., Ltd., Dalian, China); Thiazolyl 
blue tetrazolium bromide for the MTT assay (Sigma-Aldrich St. Louis, MO, USA); telomerase activity detection kit (Toyobo, Co., Ltd., Osaka, Japan).

Cell culture and transfection. Human Eca-109 and human umbilical vein endothelial ECV-304 cells (both $2 \times 10^{4}$ cells $/ \mathrm{ml}$; both purchased from Takara Biotechnology Co., Ltd.) were cultured in RPMI 1640 and Dulbecco's modified Eagle medium (both purchased from HyClone (GE Healthcare Life Sciences, Chalfont, UK) supplemented with $10 \%$ newborn calf serum with $5 \% \mathrm{CO}_{2}$ at $37.5^{\circ} \mathrm{C}$ and normal digestive subculture. Expression vectors and siRNA were transfected with Lipofectamine 2000, according to the manufacturer's protocol, and were observed under a fluorescence microscope after $24-48 \mathrm{~h}$.

Plasmid construction and siRNA. pEGFP-C3-PinX 1 and pEGFP-C3 expression vectors were synthesized in vitro by Wuhan Sanying Biotechnology (Wuhan, China) with anti-kanamycin siRNA (PinX1-FAM-siRNA sense, 5'-GUA AAGAUGUGGAAAGUUATT-3' and antisense, 5'-TTCAUU UCUACACCUUUCAAU-3'), which was used to test PinX1 expression and was composed by Shanghai Gene Pharma Co., Ltd., (Shanghai, China).

Grouping. Groupings were as follows: Group A, pEGFP-C3-PinX1 (transfected vectors with PinX1); group B, pEGFP-C3 (transfected empty vectors without PinX1); group C, Lipofectamine only; group D, control cells (Eca-109 cultured normally with no intervention); group E, PinX1-FAM-siRNA (PinX1-targeted with siRNA).

Semi-quantitative RT-PCR analysis. RT-PCR was used to analyze the expression of PinX1 in Eca-109 cells and the mRNA expression of hTERT. Following transfection for $48 \mathrm{~h}$, total RNA was extracted using TRIzol and reverse transcribed into cDNA using avian myeloblastosis virus RT. According to the manufacturer's protocol for the Takara RT-PCR detection kit (Takara Biotechnology Co., Ltd.), PinX1 mRNA was assessed using the following conditions: $94^{\circ} \mathrm{C}$ pre-denaturation ( $2 \mathrm{~min}), 94^{\circ} \mathrm{C}$ denaturation $(1 \mathrm{~min})$, followed by 25 cycles of $55^{\circ} \mathrm{C}$ annealing $(1 \mathrm{~min})$ and $72^{\circ} \mathrm{C}$ extension $(2 \mathrm{~min})$, and final extension at $72^{\circ} \mathrm{C}$ for $5 \mathrm{~min}$. hTERT mRNA was assessed using the following conditions: $94^{\circ} \mathrm{C}$ pre-denaturation $(4 \mathrm{~min})$ and $94^{\circ} \mathrm{C}$ denaturation $(30 \mathrm{sec})$, followed by 30 cycles of $49^{\circ} \mathrm{C}$ annealing (30 sec) and $72^{\circ} \mathrm{C}$ extension (45 sec), and final extension at $72^{\circ} \mathrm{C}$ for $5 \mathrm{~min}$. GAPDH was used as an internal reference for both reactions. PCR products were detected by $1 \%$ sepharose electrophoresis and were detected by a UVI Gel Imager. Quantity One software (version 4.62; Bio-Rad Laboratories, Inc., Hercules, CA, USA) was used to analyze the relative gray-scale values of PinX1/GAPDH and hTERT/GAPDH, respectively. Primers are listed in Table I.

MTT assay. An MTT assay was used to detect the proliferation of Eca-109 cells, according to the manufacturer's protocol. Eca-109 cells were inoculated in a 96-well plate, transfected and subsequently assessed for their proliferation rates after 0, 24, 48 and $72 \mathrm{~h}$ by MTT. Proliferating cells were counted using a light microscope at an absorbance value (OD) of 490 (wavelength, $490 \mathrm{~nm}$ ), and the growth curve was charted to determined the growth inhibitory rate (IR), as follows: $\mathrm{IR}=\left(\mathrm{OD} 490_{\text {control group }}-\mathrm{OD} 490_{\text {transfected group }}\right) / \mathrm{OD} 490_{\text {control }}$ group $\times 100 \%$.

Stretch PCR. Stretch PCR was used to assess telomerase activity. Eca-109 was inoculated in a 96-well plate, transfected and telomerase activity was detected after $48 \mathrm{~h}$ by stretch PCR using a TeloChaser kit (Toyobo Co., Ltd.), according to the manufacturer's protocol.

Statistical analysis. Results were analyzed by SPSS 13.0 (SPSS, Inc., Chicago, IL, USA). With the exception of cell proliferation by MTT, which was analyzed by a two-way analysis of variance (ANOVA) followed by Student-Newman-Keuls (SNP) tests, all data were analyzed by one-way ANOVA. Data were presented as the mean \pm standard deviation. $\mathrm{P}<0.05$ was considered to indicate a statistically significant difference.

\section{Results}

PinX1 mRNA expression levels. RT-PCR analysis demonstrated that there were extended PinX1 fragments in each group. pEGFP-C3-PinX1 significantly increased the expression of PinX1 mRNA in Eca-109 cells by as much as $160 \%$ $(\mathrm{P}<0.05)$. Following PinX1-FAM-siRNA transfection, the expression levels of PinX1 mRNA decreased by 70\%, which effectively silenced the expression of PinX1. No significant differences in PinX1 expression were detected in the pEGFP-C3, Lipofectamine only and control groups (Table II and Fig. 1).

pEGFP-C3-PinX1 significantly inhibits the proliferation of Eca-109 cells. Two-way ANOVA was used to analyze the proliferation rates of Eca-109 and a significant difference was detected between the groups $(\mathrm{F}=35.870$; $\mathrm{P}<0.001)$. SNK analysis demonstrated that OD490 was significantly lower in the pEGFP-C3-PinX1 group $(2.15 \pm 0.27)$, as compared with the control group $(2.52 \pm 0.50 ; \mathrm{P}=0.038)$, indicating that pEGFP-C3-PinX1 significantly inhibited the proliferation of Eca-109 cells. No significant differences in proliferation were detected between the pEGFP-C3, Lipofectamine and PinX1-FAM-siRNA groups, as compared with the control group cells, which indicated that these three treatments did not alter the proliferation of the Eca-109 cells. Furthermore, the inhibition of proliferation induced by pEGFP-C3-PinX1 was time-dependent between 24 and 48 h $(\mathrm{F}=437.621 ; \mathrm{P}<0.001)$, SNK demonstrated the OD490 value increased as the transfection duration increased from $0,24,48,72$ to $96 \mathrm{~h}$, indicating that the alteration was time-dependent. Fig. 2 demonstrates the growth curve according to the alterations in OD490 at the various time points, and the inhibition rate of proliferation was calculated. MTT assay demonstrated that pEGFP-C3-PinX1 had a significant inhibitory effect on cell growth, whereas PinX1-FAM-siRNA had no obvious effect on Eca-109.

hTERT mRNA expression. RT-PCR demonstrated that pEGFP-C3-PinX1 decreased the expression levels of hTERT mRNA by $29.9 \%$, whereas no significant difference in the expression of hTERT mRNA was detected between 
Table I. Primers of Pin 2/TRF 1 interacting protein 1 (PinX1), human telomerase reverse transcriptase (hTERT) and GAPDH for reverse transcription-quantitative polymerase chain reaction analysis.

\begin{tabular}{lllc}
\hline Gene & \multicolumn{1}{c}{ Sense (5'-3') } & \multicolumn{1}{c}{ Anti-sense (5'-3') } & Product size (bp) \\
\hline PinX1 & TTTTCTCGAGATGTCTATGCTGG & TTTTGAATTCTCATTTGGAATC & 987 \\
& CTGAACG & TTTCTTC & 670 \\
hTERT & CCGAGTGACCGTGGTTTCTGTG & GGAAGCGGCGTTCGTTGTG & 205 \\
\hline
\end{tabular}

Table II. Expression of PinX1 mRNA.

\begin{tabular}{lr}
\hline Group & mRNA \\
\hline Group A: pEGFP-C3-PinX1 & $1.599 \pm 0.102^{\mathrm{a}}$ \\
Group B: pEGFP-C3 (transfected empty vectors without PinX1) & $1.103 \pm 0.160$ \\
Group C: Lipofectamine only & $1.041 \pm 0.129$ \\
Group D: Control cells (Eca-109 cultured normally with no intervention) & $1.000 \pm 0.160$ \\
Group E: PinX1-FAM-siRNA (PinX1-targeted with siRNA) & $0.303 \pm 0.048^{\mathrm{a}}$ \\
\hline
\end{tabular}

PinX1, Pin 2/TRF 1 interacting protein 1; EGFP, enhanced green fluorescent protein; FAM, PinX1-FAM-siRNA PinX1 gene transfection by small interfering RNA. Data are presented as the mean \pm standard deviation $(n=3)$. One-way analysis of variance demonstrated $F=39.626$, $\mathrm{P}<0.001$. Pair-wise comparison demonstrated ${ }^{\mathrm{a}} \mathrm{P}<0.001$, as compared with Group D.

PinX1-FAM-siRNA, which silenced PinX1, and the control group cells ( $\mathrm{P}=0.757$; Fig. 3 and Table III).

Telomerase activity. Stretch PCR demonstrated that Eca-109 exhibited strong positive telomerase activity, whereas the ECV-304 cells exhibited weak positive activity. Following transfection, pEGFP-C3-PinX1 was able to reduce the telomerase activity of Eca-109 cells, whereas PinX1-FAM-siRNA exhibited no significant influence on the telomerase activity of Eca-109 cells. Following transfection, pEGFP C3 PinX1 was able to reduce the telomerase activity of Eca 109 cells, whereas PinX1 FAM siRNA exhibited no significant influence on the telomerase activity of Eca 109 cells. The difference between these two groups was significantly different $(F=59.436$; $\mathrm{P}<0.001$; Fig. 4).

\section{Discussion}

Eca is a type of malignant neoplasm of esophagus epithelial tissue, which is associated with a high risk (8th in the world) and mortality rate (6th in the world), as 410,000 individuals succumb to the disease each year. Eca is widespread in undeveloped countries and China is a particularly highly Eca-striken area with the highest incidence and mortality rates in the world, accounting for 210,000 Chinese lives each year. The current therapeutic strategy was not particularly effective and patients were unlikely to live longer than 5 years (1). Therefore, studies in this field have focused on the mechanism underlying Eca and the discovery of molecule-targeted treatment. In the $1990 \mathrm{~s}$, in vitro research demonstrated that the proliferation of these cells was due to telomerase activity and one of tumor cells' chief characteristics was infinite proliferation (2). This finding signaled a new era for the study of the

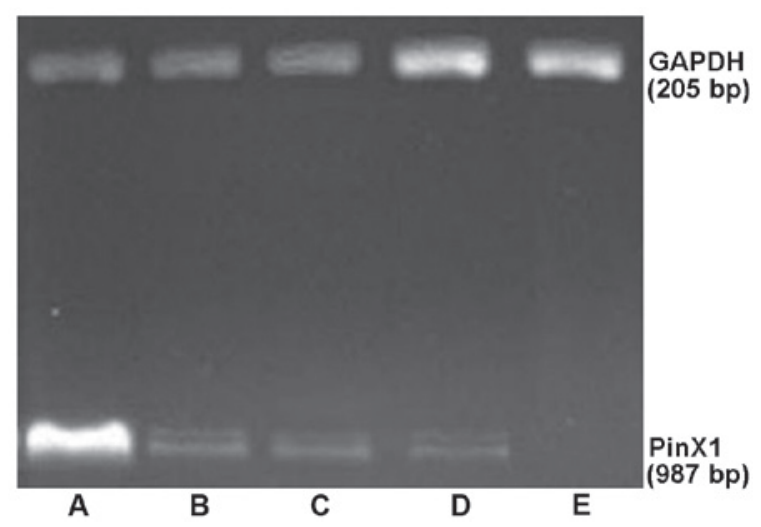

Figure 1. Expression of Pin 2/TRF 1 interacting protein 1 (PinX1) mRNA was assessed by semi-quantitative reverse transcription-polymerase chain reaction in the (A) pEGFP-C3-PinX1, (B) pEGFP-C3, (C) Lipofectamine 2000 only, (D) control and (E) PinX1-FAM-siRNA groups.

mechanisms underlying tumor cell molecules. Since telomerase has become a novel target for anti-tumor treatment, it is important to conduct further research into the control of telomerase and better cultivate its clinical value.

PinX1 is one of various conservative nucleoprotein discovered in human and yeast cells which may affect telomerase. Human PinX1 is located in chromosome 8p23, where the loss of heterozygosity often occurs in various types of cancer. Banik et al (9) analyzed the relationship among PinX1, hTERT and hTR and indicated that PinX1 was directly associated with hTERT and hTR. Furthermore, it was demonstrated that the co-work of PinX1 and hTR relied on hTERT, since PinX1 inhibition of telomerase activity was dependent upon the combination of hTERT and hTR. Chen et al (10) discovered 
Table III. Expression of human telomerase reverse transcriptase mRNA.

Group

mRNA

Group A: pEGFP-C3-PinX1

$0.701 \pm 0.088^{\mathrm{a}}$

Group B: pEGFP-C3 (transfected empty vectors without PinX1)

$0.928 \pm 0.110$

Group C: Lipofectamine only

$0.974 \pm 0.048$

Group D: Control cells (Eca-109 cultured normally with no intervention)

$1.000 \pm 0.088$

Group E: PinX1-FAM-siRNA (PinX1-targeted with siRNA)

$1.024 \pm 0.145^{\mathrm{b}}$

PinX1, Pin 2/TRF 1 interacting protein 1; EGFP, enhanced green fluorescent protein; FAM, PinX1-FAM-siRNA PinX1 gene transfection by small interfering RNA. Data are presented as the mean \pm standard deviation $(n=3)$. One-way analysis of variance demonstrated $F=5.608$, $\mathrm{P}=0.012$. Pair-wise comparison demonstrated ${ }^{\mathrm{a}} \mathrm{P}=0.003$ and ${ }^{\mathrm{b}} \mathrm{P}=0.007$, as compared with Group $\mathrm{D}$.

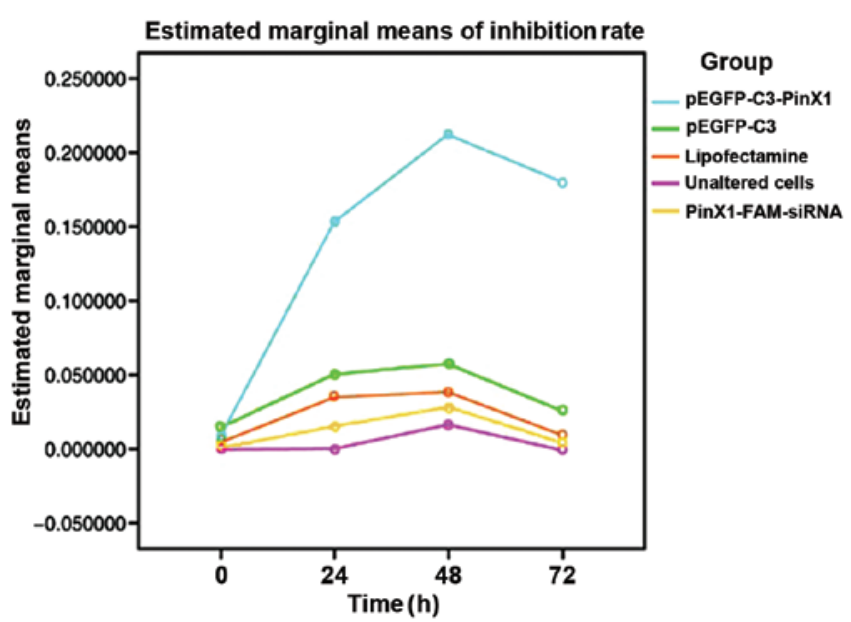

Figure 2. Inhibition rate of cell growth, as determined by MTT assay.

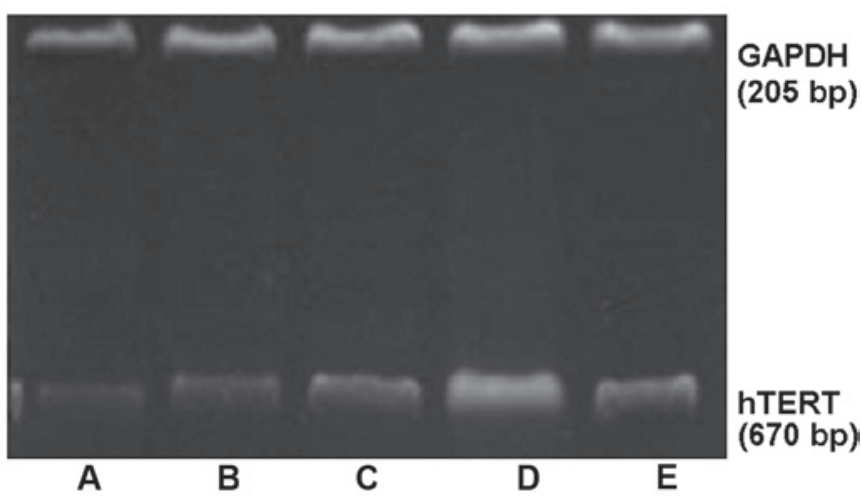

Figure 3.Expression of human telomerase reverse transcriptase (hTERT) mRNA was assessed by semi-quantitative reverse transcription-polymerase chain reaction in the (A) pEGFP-C3-PinX1, (B) pEGFP-C3, (C) Lipofectamine 2000 only, (D) control and (E) PinX1-FAM-siRNA groups.

that the mechanism of PinX1 was complex; indicating that its fragments worked respectively with Pin2/TRF1 and hTERT, LPTS/PinX1 (254-289) combined with Pin2/RF1, and in vitro LPTS/PinX1 (290-328) exhibited strong inhibition of telomerase activity and was able to shorten the telomerase to induce apoptosis. PinX1 is composed of 328 amino acids (aa), with a hTERT bonding site at both the N- (aa, 1-142) and C-terminals (aa 254-328), whereas the highly expressive

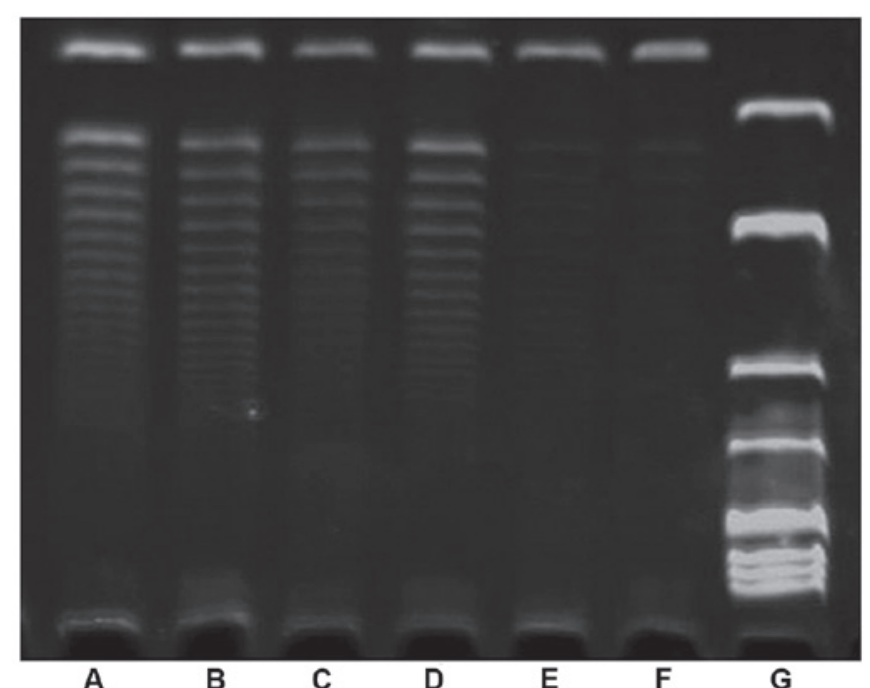

Figure 4. Telomerase activity, as assessed by stretch polymerase chain reaction in the (A) pEGFP-C3-PinX1, (B) pEGFP-C3, (C) Lipofectamine 2000 only, (D) control and (E) PinX1-FAM-siRNA groups. ECV-304 cells showed weak positive telomerase activity, whereas Eca-109 exhibited strong positive activity. These findings indicated that pEGFP-C3-PinX1 successfully inhibited telomerase activity, whereas the PinX1-FAM-siRNA, Lipofectamine 2000 only and pEGFP-C3 treatments did not alter telomerase activity.

PinX1 C fragment was able to greatly decrease telomerase activity, shorten and inhibit the growth of positive telomerase cancer cells, although it could not affect the length of negative telomerase cells $(11,12)$. Therefore, PinX1 is considered to be an effective anti-cancer gene or cancer inhibitor. In some types of cancer, such as stomach and liver cancer, studies have also demonstrated that PinX1 is able to inhibit the telomerase activity of cancer cells and induce their apoptosis $(4,5)$. However, Sun et al (13) discovered that during the differentiation of emergent acute promyelocytic leukemia, the expression of PinX1mRNA was accordant with the telomerase activity, therefore it was concluded that the expression of PinX1 was just a reaction following the expression of hTERT, aimed at maintaining stable telomerase activity. Another hypothesis proposed that in prostatic carcinoma (14), gastrointestinal carcinoma (15) and medulloblastoma (16), the expression of PinX1 had no association with telomerase activity and was not the key inhibiting factor on telomerase activity, rather just 
one phenomenon of the gene's varied states. Progress has been made in the elucidation of the dynamics of PinX1 function. Yuan et al (17) discovered that PinX1 settles in the nucleus and telomerase and would attach around chromosomes and the outside plate of the kinetochore during mitosis, during which period siRNA could be used to inhibit PinX1 and the chromosome would lose the ability to split correctly. This finding indicated that PinX1 has an important role in mitosis. Furthermore, Li et al (18) have demonstrated that, during the mitotic period, nucleolin aided the transport of PinX1 around the chromosome and enhanced the composition of the middle plate. All these studies suggested that PinX1 functions differently in various types of cancer and thus has different regulations and mechanisms. Until now, there was no unanimous hypothesis about the function of PinX1, and its underlying mechanism requires further investigation.

During the present study, an expression vector (pEGFP-C3-PinX1) and an altered RNA (PinX1-FAM-siRNA) targeted at PinX1 were successfully constructed. Following transfection of Eca-109 cells, RT-PCR was used to detect PinX1 mRNA expression levels. It was demonstrated that pEGFP-C3-PinX1 increased PinX1 mRNA expression by $160 \%$, whereas PinX1-FAM-siRNA decreased PinX1 mRNA expression by $70 \%$, which indicated that the present model of upregulation and silencing of PinX1 expression was successful, facilitating the further study of the function of PinX1 in Eca cells. The results showed that, following pEGFP-C3-PinX1 transfection of the Eca cells, both hTERT mRNA and telomerase activity were significantly inhibited (compared with the untransfected), resulting in the inhibition of the proliferation of Eca-109 cells. Following PinX1-FAM-siRNA silencing of PinX1, the telomerase activity and proliferation of the Eca-109 cells exhibited no alteration, this may be because the inhibiting function of PinX1 was hindered. It is possible that the endogenous PinX1 of Eca cells was very low or completely lacking, therefore even siRNA silencing was unable to have a marked influence on telomerase activity. Similarly, it is possible that the duration that PinX1-FAM-siRNA was able to affect PinX1 was too short (48 h), therefore it was impossible to observe the biological alteration in Eca cells.

In conclusion, the results of the present study demonstrated that the addition of exogenous PinX1 into carcinoma cells inhibited targeted telomerase activity, which indicated that PinX1 was a potential telomerase activity inhibitor. This finding may launch a novel field for treating malignant neoplasms, such as Eca, by gene-targeting.

\section{References}

1. Parkin DM, Muir CS, Whelan SL, Gao YT, Ferlay J and Powell J (eds). Cancer incidence in five continents, volume VI. International Agency for Research on Cancer, 1992.
2. Folini M, Brambil C, Villa R, Gandellini P, Vignati S, Paduano F, Daidone MG and Zaffaroni N: Antisense oligonucleotide-mediated inhibition of hTERT, but not hTERC, induces rapid cell growth decline and apoptosis in the absence of telomere shortening in human prostate cancer cells. Eur J Cancer 41: 624-634, 2005.

3. Kraemer K, Fuessel S, Schmidt U, Kotzsch M, Schwenzer B, Wirth MP and Meye A: Antisense- mediated hTERT inhibit ion specifically reduces the growth of human bladder cancer cells. Clin Cancer Res 9: 3794-3800, 2003.

4. Yu HP, Xu SQ, Lu WH, Li YY, Li F, Wang XL and Su YH: Telomerase activity and expression of telomerase genes in squamous dysplasia and squamous cell carcinoma of the esophagus. J Surg Oncol 86: 99-104, 2004.

5. Liao C, Zhao MJ, Zhao J, Jia D, Song H and Li ZP: Over-express ion of LPTS-L in hepatocellular carcinoma cell line SMMC-7721 induces crisis. World J Gastroenterol 8: 1050-1052, 2002.

6. Liao C, Zhao M, Song H, Uchida K, Yokoyama KK and Li T: Identification of the gene for a novel liver related putative tumor suppressor at a high frequency loss of heterozygosity region of chromosome 8p23 in human hepatocellular carcinoma. Hepatology 32: 721-727, 2000.

7. Sun J, Huang H, Zhu Y, Lan J, Li J, Lai X and Yu J: The expression of telomeric proteins and their probable regulation of telomerase during the differentiation of all-trans-retinoic acid-responsive and -resistant acute promyelocytic leukemia cells. Int J Hematol 82: 215-223, 2005.

8. Akiyama Y, Maesawa C, Wada K, Fujisawa K, Itabashi T, Noda Y, Honda T, Sato N, Ishida K, Takagane A, et al: Human PinXl, a potent telomerase inhibitor, is not involved in human gastrointestinal tract carcinoma. Oncol Rep 11: 871-874, 2004.

9. Banik SS and Counter CM: Characterization of interactions between PinX1 and human telomerase subunits hTERT and hTR. J Biol Chem 279: 51745-51748, 2004.

10. Chen G, Da L, Xu Y, Xu M, Song L, Li T and Zhao M: C-terminal amino acids 290-328 of LPTS/PinX1 confer telomerase inhibition. Biochem Biophys Res Commun 398: 683-689, 2010.

11. Guglielmi B and Werner M: The yeast homolog of human PinX1 is involved in rRNA and small nucleolar RNA maturation, not in telomere elongation inhibition. J Biol Chem 227: 35712-35719, 2002.

12. Lin $\mathrm{J}$ and Blackburn EH: Nucleolar protein PinX1 regulates telomerase by sequestering its protein catalytic subunit in an inactive complex lacking telomerase RNA. Genes Dev 18: 387-396, 2004

13. Sun J, Huang H, Zhu Y, Lan J, Li J, Lai X and Yu J: The expression of telomeric proteins and their probable regulation of telomerase during the differentiation of all-trans-retinoic acid-responsive and resistant acute promyelocytic leukemia cells. Int J Hematol 82: 215-223, 2005.

14. Akiyama Y, Maesawa C, Wada K, Fujisawa K, Itabashi T, Noda Y, Honda T, Sato N, Ishida K, Takagane A, et al: Human PinX1, a potent telomerase inhibitor, is not involved in human gastrointestinal tract carcinoma. Oncol Rep 11: 871-874, 2004.

15. Hawkins GA, Chang BL, Zheng SL, Isaacs SD, Wiley KE, Bleecker ER, Walsh PC, Meyers DA, Xu J and Isaacs WB: Mutational analysis of PINX1 in hereditary prostate cancer. Prostate 60: 298-302, 2004.

16. Chang Q, Pang JC, Li J, Hu L, Kong X and Ng HK: Molecular analysis of PinX1 in medulloblastom. Int J Cancer 109: 309-314, 2004.

17. Yuan K, Li N, Jiang K, Zhu T, Huo Y, Wang C, Lu J, Shaw A, Thomas K, Zhang J, et al: PinX1 is a novel microtubule-binding protein essential for accurate chromosome segregation. J Biol Chem 284: 23072-23082, 2009.

18. Li N, Yuan K, Yan F, Huo Y, Zhu T, Liu X, Guo Z and Yao X: PinX1 is recruited to the mitotic chromosome periphery by nucleolin and facilitates chromosome congression. Biochem Biophys Res Commun 384: 76-81, 2009. 